\title{
Arbor
}

\section{Memoria y relato}

\author{
José Manuel Igoa
}

Arbor CLXVII, 697 (Enero 2004), 105-123 pp.

En este artículo se hacen algunas reflexiones acerca de las relaciones entre la memoria y el relato partiendo de un intento de aclarar la semántica de estos dos términos, sobre todo del primero. En su primera parte, se examinan diversas maneras de entender y abordar estas relaciones desde una óptica predominantemente psicológica. La segunda parte del artículo se dedica a explorar el papel que desempeñan los diversos sistemas y mecanismos de la memoria humana, tal y como se conciben y estudian actualmente en la Psicología Cognitiva, en las tareas de comprender y recordar relatos. También se subrayan en ella algunos problemas relevantes que han atraido la atención de los investigadores y expertos en la materia.

\section{Sobre las relaciones entre memoria y relato}

$\mathrm{Al}$ encontrarse con un título tan escueto y genérico como el de este artículo, cualquier lector podría imaginar formas muy variadas de abordar el asunto que semejante título sugiere. Ello se debe, en buena medida, aunque no exclusivamente, al carácter polisémico del término «memoria». De hecho, el Diccionario de la Real Academia Española de la Lengua (en su $22^{\mathrm{a}}$ edición, 2001) recoge catorce definiciones de este término. Algunas de ellas nos remiten a sinónimos suyos como "recuerdo», «evocación», «rememoración» o "retentiva», palabras todas ellas que aluden, a veces indistintamente, al proceso de recordar o retener información, al producto de dicho proceso o a la capacidad, variable según indi- 
viduos y circunstancias, de recuperar los sucesos o datos que son objeto de recuerdo. Otro grupo de definiciones de la palabra «memoria» hace referencia a términos como «informe», «relación» o «exposición». Este grupo de significados se circunscribe al producto (generalmente escrito), sea cual sea el formato en que se expresa (como lista de datos, como cadena de argumentos o de sucesos, o como estructura o red de proposiciones), en el que se enumera o da cuenta de un conjunto o una serie de sucesos o datos.

Si nos ceñimos a estos dos campos semánticos en los que se sitúa el concepto de memoria y que agotan la mayor parte de sus significados, y ponemos en relación estos significados con el de la palabra «relato», podemos encontrarnos con distintas combinaciones, unas más sensatas e interesantes y otras quizá algo absurdas y redundantes. Entre estas últimas, que me apresuro a descartar para los propósitos de este artículo, está la idea de que hablar de las relaciones entre memoria y relato entraña considerar la memoria como una modalidad de relato, o al relato como el formato de redacción de una memoria. Esta idea puede parecerle al lector, muy legítimamente, fuera de lugar, sobre todo teniendo en cuenta el contexto en el que se enmarca este artículo: una revista sobre asuntos científicos y culturales que publica en el presente número una serie de ensayos que versan sobre el relato, entendido bien como género literario o bien como forma de comunicación. Quizá resultara, empero, menos descabellada si el artículo se publicara en un manual sobre métodos narrativos o sobre ciencias de la comunicación escrita. Optemos, entonces, por la interpretación a primera vista más sensata, la que nos induce a pensar que este artículo debe dedicarse a exponer una serie de reflexiones acerca de la rememoración, el recuerdo o la retención de relatos, actividades o resultados todos ellos de ejercitar la facultad de la memoria humana. La primera definición de «memoria» que encontramos en el diccionario de la RAE es enteramente coherente con esta combinación semántica de los términos «memoria» y «relato». Dice así: Memoria: facultad psíquica por medio de la cual se retiene y recuerda el pasado.

Hay un hecho gramatical, a primera vista bastante nimio, que hace más plausible la interpretación que acabo de exponer en el párrafo anterior, y es el hecho de que los términos del título vayan en singular y sin artículos, demostrativos u otras marcas lingüísticas de definitud de los nombres. Un título como «La memoria y el relato» hubiera resultado, si cabe, más ambiguo del ya de por sí ambiguo que se ha elegido para este artículo, pues hubiera permitido considerar la posibilidad de que el texto se refiriera a ejemplares concretos de una y otra categoría (de memorias y relatos) o, aún más, a dos clases de materiales textuales o infor- 
mativos pertenecientes a una categoría supraordenada más general (pongamos por caso, «la memoria y el relato ... como géneros periodisticos»).

Con todo, la interpretación elegida de la expresión «memoria y relato» va más allá de lo que escueta y literalmente dicen las palabras. La principal razón de esto es que memoria y relato desempeñan en ella papeles semánticos diferentes, en contraste con la expresión literal del título, en la que ambas palabras aparecen unidas por un nexo coordinativo como es la conjunción «y», una palabra cuyo tamaño es más pequeño de lo que denota su significado. La conjunción «y» es una partícula que expresa una relación de coordinación, en un análisis lingüístico, y representa la operación de unión en la lógica de conjuntos. No obstante, el sentido pragmático que se le debe dar en la interpretación del título del que forma parte no parece guardar relación alguna ni con su función gramatical ni con su interpretación lógica. Es, más bien, un recurso retórico que le proporciona al autor una libertad considerable para elegir la relación entre los dos conceptos de referencia que resulte más conveniente a sus intereses. Esta conjunción es como un comodín o un hueco vacío que el autor puede llevar y traer o rellenar casi a su antojo. En la interpretación favorecida aquí, «memoria» tiene un carácter predicativo y "relato» es el argumento al que se aplica dicho predicado. Según esto, parece razonable interpretar el título "memoria y relato» como «la memoria, recuerdo, rememoración, etc. (que tenemos o nos formamos) de un relato", expresión esta en la que la memoria aparece como una suerte de cualidad o atributo del relato o, mejor aún, como una operación a la que se le somete.

Pero incluso si aceptamos esta primera definición de «memoria» reseñada en el diccionario, enseguida se nos revelan distintas maneras de abordar la cuestión mencionada en el título, según sea el énfasis que uno quiera poner en cada uno de los elementos de la definición. Así, una alternativa supondría optar entre la consideración de la memoria como facultad psíquica (o mental, por utilizar una expresión más acorde con los usos psicológicos actuales) y la idea de memoria como receptáculo o almacén de recuerdos acerca del pasado. Ambas nociones están incluidas en la definición. Sin embargo, el tratamiento de las relaciones entre la memoria y el relato que se emprenda bajo una u otra óptica con seguridad resultará distinto en cada caso. En el primero, la memoria desempeña un papel activo, destacando como conjunto de procesos u operaciones mentales con un papel causal (o al menos funcional) en la generación y en la comprensión de relatos y narraciones. Desde esta perspectiva, se tenderá a subrayar la contribución de la memoria, en sus distintos com- 
ponentes y sistemas, a la interpretación y composición de historias narradas. En el segundo caso, la memoria aparece insinuada como resultado o producto, como estructura de información que queda almacenada de las narraciones o relatos leídos o escuchados en el pasado. En resumen, la memoria se puede considerar como causa o como efecto de la actividad de producir y escuchar relatos, y preferiblemente como ambas cosas a la vez. En un sentido relacionado, el relato se puede considerar, a su vez, como una de las formas, quizá la forma por excelencia, de articular y revelar (y tal vez también de representar permanentemente) los contenidos de nuestra memoria, esto es, nuestros recuerdos, sean éstos autobiográficos, episódicos o abstractos. Si echamos una mirada hacia los textos de psicología de un pasado no muy remoto que se refieren a esta cuestión, comprobaremos que el enfoque de la memoria como elemento causal atrae más atención en los textos más recientes (véase por ejemplo, el de Elosúa, 2000), mientras que el tratamiento de la memoria como receptáculo de almacenamiento y representación del conocimiento es más típico entre los textos más antiguos (por ejemplo, Clark y Clark, 1977).

Aún queda otro par de detalles, no menos importantes, para completar esta reflexión preliminar sobre la relación entre memoria y relato. El término "relato» no es, desde luego, tan prolífico en significados como el de «memoria», aunque su significado extensional (es decir, el conjunto de objetos al que puede aplicarse el término) puede ser variable. En términos generales, «relato» es palabra sinónima de «narración» o «cuento», es decir, la expresión de un conjunto organizado de sucesos o episodios acaecidos o imaginados. Se trata, además, de un concepto que se solapa con el de texto o discurso, en la medida en que un relato puede ser una narración escrita (como texto) o hablada (como discurso), aunque no tiene por qué agotarse en estos formatos de expresión, ya que también puede incluir imágenes estáticas o en movimiento. Por ello, cabe decir que no todo relato es, únicamente y a la vez, texto o discurso. Y a la inversa, si se insiste en su carácter narrativo, es obvio que hay textos y discursos no narrativos, y que por tanto el relato es, todo lo más, un género o una variedad del discurso oral o del texto escrito. Si volvemos al diccionario de la RAE como criterio de corrección semántica, encontramos un aval a estas observaciones informales. Las definiciones de «relato» que allí aparecen son dos (una, en realidad, pues la segunda es una simple enumeración de sinónimos): (1) conocimiento que se da, generalmente detallado, de un hecho; y (2) narración, cuento. La primera definición manifiesta de forma sutil el doble carácter del concepto en cuestión: por un lado, el hecho de que se trata de conocimiento, esto es, de información representada de forma permanente; y por otro, el hecho de que es un conocimiento 
transferible o comunicable, con independencia del soporte o modalidad elegida para comunicarlo. La segunda definición revela el carácter narrativo o episódico que a menudo (aunque no siempre, según se desprende de la primera definición) se atribuye a los relatos.

De las definiciones de memoria y relato mencionadas anteriormente y de sus consecuentes relaciones se desprende el enfoque que adoptaré en este artículo para el tratamiento de tan complejo asunto. Sin embargo, aún me queda por hacer una última disquisición para pergeñar del todo este enfoque. Cuando se dice que el relato o la narración constituyen para los humanos formas naturales de almacenar, organizar y representar información o conocimientos, se pueden adoptar aún dos puntos de vista diferentes según quién se suponga que es el depositario de dicha información o conocimiento. Si se adopta el punto de vista de la psicología individual o personal, tal y como se hace en la llamada Psicología Cognitiva, corriente dominante hoy en día en la psicología que lleva el marchamo de "científica», se pondrá el énfasis en los procesos que lleva a cabo la mente cognitiva en la comprensión y el recuerdo de narraciones o relatos. Desde esta óptica, el problema que ha de ser objeto de explicación (el explanandum) es la comprensión y el recuerdo de relatos, mientras que la solución que explica el problema (el explanans) son los recursos y procesos de la memoria de que dispone la mente individual y que se ponen en funcionamiento ante la tarea de interpretar un relato o de recuperar el conocimiento que se tiene del mismo. La segunda parte de este artículo estará dedicada a examinar la contribución de la memoria, entendida como facultad de la mente individual (tal y como veíamos en la definición del diccionario) al procesamiento de relatos y narraciones.

Pero hay otra forma de abordar el asunto. Si consideramos la memoria más como un producto que como un proceso, podemos desplazar nuestro punto de mira desde el ser humano individual hacia las sociedades o grupos humanos como entidades depositarias de una memoria colectiva, memoria que organiza y mantiene los recuerdos compartidos por los miembros del grupo social a través del tiempo. Esta forma de memoria tiene como una de sus funciones primordiales la de crear un vínculo de identificación o un sentido de pertenencia del individuo a una colectividad. Hay diversas formas de crear y mantener la memoria colectiva de un grupo humano y distintos soportes para realizar estas tareas. En la siguiente cita, tomada de la reseña de un libro publicado recientemente, se resumen las principales ideas de esta perspectiva.

No hay identidad sin memoria. Esta es una afirmación válida tanto para los individuos como para los grupos y, desde luego, para las naciones. La Historia es una de las formas de conservar la memoria de una sociedad, pero no es la única forma de re- 
presentarse, o de imaginar, el pasado y preparar el futuro. Monumentos, rituales, obras literarias y otras actividades culturales son también medios para el recuerdo y el olvido. Las emociones y las formas de poner en palabras los acontecimientos vividos se dan en la comunicación próxima, en los discursos públicos y en la enseñanza. Sobre estos procesos descansa tanto la constitución de los eventos memorables, como la producción de recuerdos y la articulación de futuros deseables o temidos. (Rosa et al., 2002).

Aunque el énfasis del libro reseñado en esta cita se pone en el papel de la memoria colectiva en la creación de un sentido de identidad nacional, las observaciones contenidas en ella valen igualmente para cualquier otra forma de identidad individual, grupal o transnacional. Por otra parte, de esta cita pueden extraerse dos consideraciones de interés para entender mejor la orientación que defiende. En primer lugar, parece obvio que la noción de memoria que se expresa en ella está poco vinculada a la idea de memoria que se maneja en psicología cognitiva. La única relación aparente se puede encontrar en la idea de una memoria autobiográfica, una variante de la memoria episódica en la que se representan sucesos relevantes de la vida o de la historia de personas, grupos sociales, etnias, naciones, culturas o civilizaciones. A esta forma de memoria, la episódica, es a la que implícitamente se alude en la definición del diccionario que está sirviendo de hilo argumental en este texto. $\mathrm{Su}$ función, pues, es la de "recordar», «retener», «evocar» o «rememorar» sucesos, verbos que denotan el proceso de recuperación de información narrativa que está en la base de esta facultad. En segundo lugar, es importante resaltar que la memoria colectiva es una memoria compartida, que se mantiene en los miembros de la colectividad a través de fenómenos públicos o soportes externos que permiten la comunicación o el intercambio de experiencias recordadas o elaboradas en el relato histórico. El principal soporte externo para compartir la memoria colectiva es, sin duda alguna, el lenguaje, aunque como se dice en la cita, éste no sea el único. El lenguaje es la materia fundamental de la que están hechos los relatos y es también el vehículo idóneo para transmitirlos en la conversación privada, en los actos públicos y en la instrucción formal. Dando un paso más, podemos incluso decir, de acuerdo con Vygotski, que el lenguaje es la herramienta capital para constituir la conciencia individual, en tanto en cuanto pone al alcance del sujeto los signos públicos que, a través de un proceso de interiorización, terminan por configurar su mundo de significados privados.

$\mathrm{Al}$ igual que el lenguaje articulado, la narración, como forma de representación y expresión de la memoria colectiva, es un fenómeno típicamente, si no exclusivamente, humano. Lenguaje y narración confieren a 
la memoria episódica, que compartimos con otros animales, un carácter distinto, al permitirle acceder a un sistema de conocimiento, una nueva forma de memoria que los psicólogos han dado en llamar memoria semántica. En palabras de Endel Tulving, que fue quien propuso por primera vez esta distinción entre estos dos sistemas de memoria, episódica y semántica, la memoria semántica «permite a los animales, humanos y no humanos, adquirir y utilizar conocimiento acerca de su mundo» (Tulving, 2002). Como señala este autor, mientras que la memoria episódica se ocupa de recordar, la memoria semántica se ocupa de conocer. Así pues, si tomamos el relato como objeto lingüístico, vemos que el lenguaje no sólo debe concebirse como objeto de rememoración, como resultado del ejercicio de la memoria colectiva, sino que también debe considerarse un factor esencial en la constitución y el mantenimiento de los recuerdos. Visto de este modo, el lenguaje nos faculta para desarrollar una forma narrativa de pensamiento, en la expresión empleada por Bruner (1986), que se define como la capacidad de construir descripciones de eventos y objetos y razonar sobre ellos. En un libro muy sugerente, Merlin Donald (1991) propone la idea de que la evolución de las capacidades cognitivas ha atravesado tres grandes fases en la transición de los primates pre-homínidos al homo sapiens moderno. Estas tres etapas corresponden a otras tantas formas de pensamiento y de cultura que corren paralelas al desarrollo de la capacidad simbólica en los primates superiores, a saber, la cultura episódica, la cultura mimética y la cultura mítica.

La cultura episódica, según Donald, caracteriza a algunas especies de mamíferos, incluidos los primates no humanos y probablemente los primeros ancestros del linaje homo. Se trata de una cultura ligada al presente, a los episodios y sucesos concretos que acontecen en el entorno inmediato. Esta sujeción a situaciones inmediatas y concretas hace que su comportamiento dependa de un sistema de memoria comparativamente rudimentario, como es la memoria episódica, y su aprendizaje esté basado en la observación y el recuerdo de situaciones específicas. La actividad simbólica apenas se encuentra desarrollada y la transmisión de prácticas culturales es muy limitada. No obstante, en este nivel de desarrollo cultural aparecen algunos indicios de conciencia individual y de capacidades de reconocimiento de intenciones, lo que presupone un desarrollo simbólico incipiente. La cultura mimética descansa en la capacidad de producir actos conscientes y voluntarios con carácter intencional. Esta cultura, que Donald atribuye a los homínidos prehumanos (como el homo erectus), supone ya un mayor grado de abstracción, un distanciamiento de la realidad inmediata y, por tanto, una capacidad de anticipar situaciones 
(pensamiento hipotético). La conducta mimética va más allá de la simple imitación, pues supone la capacidad de recrear gestos y acciones de forma desplazada y con valor intencional y tiene además un carácter combinatorio, esto es, es capaz de descomponer las acciones en sus elementos constitutivos y recombinar estos componentes para crear acciones novedosas. Sin embargo, el pensamiento mimético continúa, por así decirlo, prisionero de la memoria episódica, en la medida en que no pasa de ser un pensamiento reconstructivo basado en el recuerdo del pasado. La cultura mítica aparece con el advenimiento del homo sapiens moderno, heredando las características de la cultura mimética e imprimiéndoles un nuevo giro. Es una cultura de la invención. Con ella aparecen los mitos, narraciones que se constituyen en modelos conceptuales del mundo y que facilitan la expansión de las capacidades simbólicas. En esta etapa de desarrollo del pensamiento, la memoria episódica, fundamento de las formas de pensamiento precedentes, se ve esencialmente enriquecida y modificada por la memoria semántica. En este sistema de representación del conocimiento, la información está codificada en un formato abstracto (lingüístico o proposicional). De hecho, gracias a los rasgos de arbitrariedad y desplazamiento, el lenguaje es un vehículo idóneo para representar información de manera abstracta, por lo que cabe suponer (como sostiene Donald) que la aparición del lenguaje en la escena evolutiva fuera un factor indispensable para el desarrollo de la cultura mítica.

Resumiendo: el relato es una forma de representación y comunicación de nuestra experiencia presente y pasada (y una anticipación de la futura). Es un instrumento diseñado para configurar nuestra memoria individual y colectiva que hunde sus raíces en el pensamiento narrativo, una capacidad arraigada desde muy antiguo en nuestra especie que se sostiene sobre dos sistemas de memoria complementarios, la memoria episódica y la memoria semántica. El modo en que se complementan estos dos sistemas de memoria será objeto de reflexión en la segunda parte de este artículo. En ella desarrollaré también algunas ideas esbozadas en esta primera parte acerca del papel que desempeñan otros sistemas de memoria en los procesos de comprensión de narraciones.

\section{Procesos y sistemas de memoria en la comprensión de relatos}

En este apartado haré un breve esbozo de los sistemas de memoria que han venido proponiéndose en las diversas teorías de la psicología contemporánea. Sobre este mapa iré dibujando los procesos y recursos de memoria que aparecen implicados en la tarea de comprensión de textos 
y señalando, de paso, algunas cuestiones que son objeto de debate en la investigación actual.

La distinción establecida en la primera parte del artículo entre memoria episódica y memoria semántica se puede completar ahora señalando que estos dos sistemas de memoria se concibieron desde un principio como dos variantes de un tipo de memoria que se ha dado en llamar «declarativa», toda vez que ambas se definen como almacenes permanentes de información. Con ello, se distinguen de otros sistemas de memoria que cumplen una función orientada a procesar la información, esto es, a efectuar operaciones de codificación, transformación y recuperación de los datos que entran en nuestra memoria o se recuperan de ella con diversos fines. Estos sistemas de memoria, diseñados para el tratamiento de información, se encuadran bajo el rótulo de «memoria procedimental», y guardan una estrecha relación con los procesos de la memoria operativa, de los que hablaré más tarde. Los dos sistemas de la memoria declarativa, el episódico y el semántico, presentan ciertas características comunes que resulta oportuno mencionar aquí (cf. Tulving, op. cit.). Así, ambas pueden codificar información en formatos diversos (visual, lingüístico, proposicional), ambas permiten (aunque no tiene por qué exigir) un acceso por introspección a la información que contienen y, lo que es más importante para los propósitos del tema que nos ocupa en este artículo, ambas contienen información que es semánticamente evaluable, es decir, que tiene valor de verdad. Nuestras creencias acerca del mundo y nuestros recuerdos acerca de los sucesos vividos o imaginados pueden ser verdaderos o falsos. Esta propiedad de los contenidos de la memoria tiene unos efectos paradójicos sobre la comprensión y el recuerdo de relatos, ya que aunque el valor de verdad constituya un rasgo definitorio de los contenidos de nuestra memoria, el modo en que operamos con esos contenidos al elaborarlos, comprenderlos y razonar sobre ellos en el procesamiento del relato es, por lo común, bastante ajeno al hecho de que sean ciertos o inciertos. Por una parte, es un hecho conocido que el recuerdo (y la comprensión misma) de un relato suele comportar una distorsión y una reconstrucción considerables de la información contenida explícitamente en él. No sólo olvidamos de inmediato los detalles lingüísticos que se expresan en la superficie del texto (una estrategia muy frecuente que emplean los autores para mostrar directamente este efecto es el de pedir al lector que trate de recordar textualmente la última frase que han leído en el texto), sino que añadimos, a veces de forma innecesaria e incluso perjudicial para la adecuada comprensión del mismo, información que el texto no contiene. Más allá de la información dada es el título de una obra ya clásica de la psicología cognitiva que se repite en muchos manuales sobre la materia para expresar este hecho. Esta prác- 
tica de ir más allá de la información dada ocurre de forma regular durante la comprensión del lenguaje en general, y a pesar de los riesgos de error que entraña, a la postre resulta eficaz y oportuna de cara a la comprensión de textos, pues si sopesamos sus ventajas e inconvenientes, las primeras sobrepasan ampliamente a los segundos. Desde un punto de vista formal, se trata de un proceso de razonamiento deductivo del que se extraen inferencias a partir de premisas, que se añaden implícitamente a la información del texto y que se obtienen del conocimiento general del lector. Más adelante volveré sobre el asunto de las inferencias en la comprensión de textos, que es, sin lugar a dudas, uno de los temas que más debates ha suscitado entre los investigadores.

Un segundo efecto paradójico del valor de verdad de los relatos en la comprensión y el recuerdo de los mismos es que, en muchas ocasiones, el hecho de que el relato cuente hechos verdaderos o falsos resulta del todo irrelevante. Una notable excepción deberían ser los relatos de prensa, aunque en la práctica, muchas veces ni siquiera se les exige un mínimo compromiso con la verdad. En cuanto a los relatos históricos, hay pocos de los que se presuponga ese compromiso con la verdad. Los relatos, en general, no tienen por qué contar hechos verdaderos y una de las razones de ello es que no representan hechos directamente, sino creencias acerca de los hechos que cuentan. En otras palabras, los relatos son metarrepresentaciones del mundo ${ }^{1}$. Por este motivo, se puede decir de ellos, como de cualquier metarrepresentación, que son conjuntos de enunciados referencialmente opacos, por usar la expresión de Quine (1960). La opacidad referencial es una propiedad que exhiben algunos enunciados que impide mantener ciertos compromisos ontológicos con otros enunciados sinónimos, como el de la preservación de su valor de verdad. Así, por ejemplo, un sujeto puede creer verdadero un enunciado (Juan está bebiendo agua) y falso un sinónimo del mismo en el que se han sustituido los argumentos (Un chico rubio está bebiendo $\mathrm{H}_{2} \mathrm{O}$ ) sin incurrir en contradicción. Si queremos darle un aire algo más grandilocuente a estas observaciones, podríamos decir que el relato es una herramienta muy apropiada para despegarnos de la realidad inmediata y crear y compartir mundos imaginarios. $\mathrm{Y}$ todo ello sin correr el riesgo de confundir lo real con lo imaginado, ya que la capacidad de construir metarrepresentaciones del mundo lleva implícita la de mantener las metarrepresentaciones separadas de las representaciones sobre las que versan a la vez que se conserva intacta la relación entre ellas (Leslie, 1987).

Otra cuestión importante en la discusión sobre el papel de los sistemas de memoria episódico y semántico en la comprensión de relatos es la de la relación que guardan entre sí estos dos sistemas de memoria (y sus 
respectivos contenidos) durante los procesos de comprensión. Dicho de otro modo, ¿cómo intervienen los conocimientos semánticos y episódicos en la formación de representaciones acerca de los contenidos del relato? Para responder a esta pregunta es preciso examinar el papel de la memoria operativa en estos procesos.

Como señala Tulving (op. cit.), la memoria episódica aparece después de la semántica, tanto en la historia evolutiva como en el desarrollo humano. Como ya he mencionado anteriormente, la memoria episódica de nuestros antepasados más próximos es la artífice del pensamiento narrativo. Sin embargo, una memoria episódica capaz de representar hechos del pasado tiene que asentarse por fuerza en una base de conocimiento permanente sobre los objetos, los individuos, las cualidades y las relaciones que existen en nuestro entorno. Por ello, la memoria episódica no es sólo filogenéticamente posterior a la memoria semántica, sino que la presupone lógicamente. La construcción narrativa de sucesos y episodios significativos sólo puede darse si disponemos de representaciones duraderas de los conceptos que participan en tales sucesos. El asiento de estas representaciones es la llamada memoria a largo plazo (MLP), que más que un sistema de memoria en sentido estricto es un estado de nuestros conocimientos del mundo. Los contenidos de la memoria a largo plazo se hacen presentes a la conciencia de forma transitoria en la memoria a corto plazo (MCP), que tampoco debe considerarse un sistema separado e independiente de la memoria, sino el estado de activación momentáneo de un fragmento limitado de información (que el psicólogo George Miller llegó a cuantificar en $7 \pm 2$ unidades de información) que cae bajo el foco de la atención mientras es objeto de procesamiento.

La descripción tradicional del funcionamiento integrado de la MLP y la MCP se atenía a un esquema secuencial, en el cual la información que entraba en el sistema cognitivo a través de los sistemas perceptivos pasaba primero por un almacén transitorio, la MCP, que tras una serie de ensayos transfería esta información a la MLP, donde quedaba permanentemente almacenada. Este esquema simplificado fue dando paso a otros modelos algo más complejos, que trataban de dar cuenta de ciertos efectos experimentales que el esquema original dejaba sin explicar. Uno de estos efectos es el llamado efecto de niveles, que voy a describir aquí debido al interés que presenta para los procesos de comprensión de textos y discursos. En su formulación general, el efecto de niveles parte de la distinción de varios niveles de profundidad en la codificación de la información en la memoria. En el caso de los textos, y prescindiendo de los niveles más locales de organización de la información (fonológico, morfológico, léxico o sintáctico), el procesamiento discurre desde niveles más 
superficiales, como el formado por la red de proposiciones que lo constituyen y del que se extrae el «texto base», hasta otros niveles más profundos y abstractos como el de la «macroestructura», que incluye las proposiciones que se sitúan en un plano jerárquicamente superior y representan las ideas principales del texto. El efecto de niveles consiste en la mayor probabilidad de retener en MLP aquellas proposiciones que pertenecen a la macroestructura del texto en perjuicio de las proposiciones de niveles inferiores (Kintsch y Van Dijk, 1978). Esto, en definitiva, no hace más que consagrar la idea intuitiva de que tendemos a recordar mejor las ideas principales de un texto. Sin embargo, según lo descrito hasta aquí, los procesos de codificación en MLP de la información de un texto aparecen como procesos lineales y unidireccionales, que abstraen representaciones generales a partir de la información particular contenida en el propio texto. Pero como ya he indicado anteriormente, esta concepción pasa por alto el hecho de que la comprensión de textos es también un proceso guiado conceptualmente, dirigido por los conocimientos y las expectativas del lector o el oyente, y, en particular, ignora el conocimiento de que la comprensión del un texto es un proceso reconstructivo que va más allá de la información expresamente contenida en él.

El concepto de memoria operativa (también conocida como «memoria de trabajo», por traducción de la expresión inglesa working memory) es heredero del de memoria a corto plazo. Este sistema de memoria, que en su origen fue concebido como un almacén transitorio de la información en el camino hacia su almacenamiento permanente en MLP, sufrió una profunda modificación que llevó a convertirlo en una forma de memoria mixta (procedimental y declarativa), cuyo cometido era no sólo el de recuperar temporalmente la información pertinente para efectuar ciertas tareas de resolución de problemas, sino también el de realizar operaciones de cómputo sobre esa información. Hoy en día, la memoria operativa se considera un componente esencial de procesamiento en prácticamente cualquier tarea cognitiva mínimamente compleja. En su formulación original (cf. Baddeley y Hitch, 1974), la memoria operativa se consideraba un único sistema de memoria con varios componentes: un sistema ejecutivo dedicado al control de la atención y dos sistemas subsidiarios encargados de retener información específica de dominio (la «agenda visoespacial», para la información visual, y el «bucle fonológico» para la información verbal). Actualmente se piensa que puede haber memorias operativas especializadas en dominios separados (verbal y espacial, al menos), cada una de ellas con un componente de procesamiento y otro de almacenamiento que funcionan de forma coordinada. El hallazgo de pacientes con lesión cerebral que presentan alteraciones selectivas de la 
memoria operativa en un dominio mientras mantienen intacta su memoria operativa en otro distinto ha reforzado considerablemente esta visión «modular» de la memoria operativa.

Examinemos ahora en el papel de la memoria operativa verbal en tareas de comprensión de textos narrativos. Uno de los hallazgos más conocidos al respecto es que existe una correlación entre la amplitud de esta memoria (cuantificada en términos de la capacidad de retener la última palabra de cada oración de una secuencia de oraciones -de longitud variable- que forman un texto) y el rendimiento en distintas tareas de comprensión de textos (como por ejemplo, la identificación de antecedentes pronominales o el recuerdo de proposiciones). Las diferencias de rendimiento observadas entre individuos de elevada y de baja amplitud de memoria se atribuyen a la eficacia con que se gestionan los recursos de procesamiento y de almacenamiento, y no simplemente a la capacidad de almacenamiento de información en MCP en términos absolutos (Gathercole y Baddeley, 1993). Los estudios relativos al papel de la amplitud de memoria en el procesamiento de textos se centran, por lo general, en procesos relativamente locales, que entrañan la integración a corto plazo de información léxica en estructuras proposicionales. Se trata de procesos que utilizan información explícitamente representada en el texto y cuya integración depende más de las relaciones gramaticales y semánticas que se dan entre distintos fragmentos del mismo que de información implícita, ajena al texto, que el lector deba invocar para comprenderlo.

Un tipo diferente de procesos implicados en la comprensión de narraciones son aquellos que requieren el uso inmediato de información declarativa representada en la memoria a largo plazo. Estos procesos, a diferencia de los mencionados en el párrafo anterior, operan de arriba hacia abajo a partir de ciertas claves presentes en el texto, como por ejemplo, palabras de contenido. La presencia de estas claves en la memoria operativa a corto plazo desencadena un proceso de activación automática de una parte de los contenidos de la MLP. Esta activación acontece de un modo automático, toda vez que dichas claves tienen unos vínculos estables con unidades de representación de la MLP. La estructura de memoria activada de este modo se ha denominado memoria operativa a largo plazo (Ericsson y Kintsch, 1995). Este sistema de memoria no adolece de las limitaciones de capacidad que caracterizan a la memoria operativa a corto plazo y, según los autores que lo han propuesto, se manifiesta en ciertos dominios de conocimiento experto. El proceso de activación de representaciones en la memoria operativa a largo plazo se ha descrito como un proceso en dos partes: de un lado, debe darse una activación automática de una red de nodos léxicos asociados con las palabras clave del tex- 
to; de otro, debe producirse un proceso de desactivación de los nodos que sean irrelevantes para el significado contextualmente apropiado del enunciado. Así, por ejemplo, ante una oración como «El gabinete estudia medidas para evitar la fuga de cerebros», prácticamente todas las palabras de contenido («gabinete», «estudia», «medidas», «fuga» y «cerebros») poseen términos asociados relevantes e irrelevantes para una adecuada interpretación. Entre los primeros podrían hallarse palabras como «gobierno», «examina», «decisiones», «emigración» y «expertos», y entre los segundos, palabras como «habitación», «aprende», «dimensiones», «huida» y «cráneo». La desactivación de este segundo grupo de términos asociados es indispensable no sólo para la correcta comprensión del enunciado, sino también para que éste pueda generar las inferencias adecuadas para la comprensión de las sucesivas partes del texto. Nótese que la selección de los términos apropiados y la supresión de los inapropiados no puede guiarse exclusivamente por la frecuencia de uso de los significados de las palabras. Por ello, el proceso de activación automática de nodos léxicos se lleva acabo a partir de un cálculo de las «co-ocurrencias» de las palabras pertinentes en extensos corpora de material escrito. Este procedimiento ayuda a mitigar la proliferación de asociaciones indeseadas que podrían bloquear el proceso de comprensión del enunciado. Si bien para Ericsson y Kintsch el proceso de activación es automático, el proceso complementario de desactivación que le sigue parece tener un carácter más estratégico, es decir, probablemente queda bajo el control voluntario del sujeto y está supeditado a los objetivos del lector.

Las últimas observaciones que he realizado en torno a la memoria operativa a largo plazo nos sitúan ante uno de los problemas que más interés ha suscitado entre los investigadores de los procesos de comprensión de textos, el de la naturaleza y el papel de las inferencias en la comprensión. El término «inferencia» se emplea en el campo de la comprensión de textos y discursos en un sentido distinto, aunque relacionado, al que se usa en lógica. En sentido lógico, la inferencia es la operación por la que se extraen conclusiones o consecuencias de unas premisas. Esta operación se realiza mediante unas reglas bien definidas. En el procesamiento de textos, la inferencia se entiende bien como la operación que permite obtener información no explícitamente representada en un texto o como la información inferida a través de dicha operación. Bien es verdad que inferir información no explícita en un texto supone, en cierto modo, extraer conclusiones a partir de unas premisas. El problema está en que no siempre se establece con claridad cuáles son estas premisas o cómo se hallan representadas, y mucho menos cuáles son las reglas que los sujetos emplean en el proceso, suponiendo que las operaciones de in- 
ferencia textual estén basadas en reglas. Por ello, en este contexto, la inferencia parece más un término de conveniencia para denotar cualquier operación que implique añadir información a un texto al comprenderlo o al recordarlo. Quizá por ello han proliferado las taxonomías de inferencias textuales, según fuera el criterio de clasificación privilegiado.

Entre los criterios que se siguen para clasificar las inferencias realizadas en la comprensión de textos figuran el objeto de las inferencias, el contenido de las mismas, la dirección en que se efectúan, su grado de automatismo u obligatoriedad, el momento del procesamiento en que tienen lugar y el tipo de procesos que se emplean en su realización. Atendiendo a su objeto, las inferencias pueden ser semánticas, cuando se refieren a algún aspecto del contenido proposicional del discurso, es decir, a hechos implicados o no expresados directamente en el texto que se pueden deducir del conocimiento general del oyente o lector, o pragmáticas, cuando conciernen a las intenciones o estados mentales del hablante o escritor o de los personajes que intervienen en el relato. En función de su contenido, las inferencias pueden ser de muy diversos tipos: causales, cuando establecen relaciones de causalidad entre hechos o proposiciones del relato (véase el ejemplo 1 más abajo), consecutivas, cuando anticipan posibles consecuencias de los hechos que se relatan (ejemplo 2), instrumentales, cuando fijan la identidad de objetos no mencionados en el relato que intervienen como instrumentos de acciones (ejemplo 3), o anafóricas, empleadas para localizar (ya sea dentro ô fuera del discurso) el antecedente de una expresión lingüística (como los pronombres personales en cursiva del ejemplo 4). (En los ejemplos se añaden preguntas que ponen de relieve el objeto de la inferencia.

(1) Juan se asomó a la ventana alarmado. El ruido de sirenas era ensordecedor. (¿Por qué se asomó Juan a la ventana?)

(2) María olvidó meter las cervezas en la nevera. Cuando más tarde llegaron sus amigos, les ofreció algo de beber. (¿Cómo estaban las cervezas cuando llegaron los amigos?)

(3) El jardinero estuvo toda la mañana podando los rosales de la marquesa. (¿Qué usó el jardinero para podar los rosales?)

(4) Jorge fue a visitar a Julián para devolverle el dinero que le debía. (¿Quién iba a devolver/debía dinero a quién?)

Según la dirección en que se realizan, las diferencias pueden ser predictivas, si anticipan hechos aún no narrados, o retrospectivas si se usan para elaborar o completar hechos ya mencionados en el relato. En otro orden de cosas, las inferencias pueden realizarse de forma automática, sin que el oyente o lector se lo proponga de forma consciente, o estratégica, 
es decir, bajo control voluntario; y con independencia de esto último, algunas inferencias se consideran obligatorias para la comprensión del relato y otras constituyen meros adornos o elaboraciones de la información, sin que sean indispensables para la comprensión. Por lo que respecta a su curso temporal, algunas inferencias ocurren de forma rápida e inmediata, o en fases relativamente tempranas del proceso de comprensión, y otras se elaboran más lentamente y en etapas más tardías del mismo; incluso se supone que hay inferencias que se construyen a posteriori, en el momento de evocar o rememorar ciertos episodios del relato. Por último, las inferencias pueden llevarse a cabo a través de diversos tipos de procesos, desde procesos de razonamiento basado en reglas hasta procesos de asociación entre piezas léxicas que se emplean en la narración. (Una reciente y pormenorizada revisión de la cuestión de las inferencias en la comprensión de textos se puede encontrar en León, 2003).

Como el lector podrá fácilmente comprender, estos criterios, y las clasificaciones resultantes de su aplicación, no tienen por qué ser siempre independientes unos de otros. Por ello, es posible definir perfiles o modalidades de inferencia en función del valor que se atribuya a cada uno de los criterios referidos. Por ejemplo, cabe suponer que las inferencias obligatorias sean al mismo tiempo rápidas y automáticas y se efectúen mediante mecanismos de asociación léxica. Por el contrario, parece razonable pensar que las inferencias basadas en reglas sean comparativamente más lentas, estratégicas y a menudo opcionales. Sin embargo, aún quedan bastantes incógnitas por despejar en materia de tipología y procesamiento de inferencias en la comprensión. Voy a detenerme a comentar una de las cuestiones a mi entender más relevantes al respecto.

Uno de los asuntos más debatidos entre los investigadores de este campo es hasta qué punto la realización de inferencias es una actividad frecuente y regular durante la comprensión de textos o, por el contrario, se trata de una actividad relativamente excepcional y restringida. La primera opción es la que defienden los modelos constructivistas como el de Graesser y colaboradores (Graesser y Kreuz, 1993), mientras que la segunda caracteriza al modelo minimalista (McKoon y Ratcliff, 1992). Los modelos constructivistas conciben el proceso de comprensión como un proceso guiado conceptualmente en el que el lector crea un modelo de la situación descrita en el texto a partir de su conocimiento previo. Este modelo de situación se enriquece y modifica conforme avanza la lectura a partir de la continua realización de inferencias. El objetivo principal de los modelos constructivistas es dar cuenta de la construcción de una representación global y coherente del texto. Y este empeño parece depender más de los objetivos del lector que de las características del texto. En 
cambio, el modelo minimalista hace más hincapié en los procesos implicados en la búsqueda de coherencia local. Por ello reduce las inferencias al mínimo imprescindible para enlazar las partes del texto que carecen de vínculos explícitos, poniendo como restricción fundamental que las inferencias generadas sean rápidas y poco costosas en cuanto a los recursos de memoria necesarios para su realización. Como es habitual en muchas áreas de la psicología cognitiva, este debate no parece que tenga visos de solución a corto plazo. Como han señalado varios críticos, estos dos modelos no sólo difieren en su manera de entender el proceso de comprensión, sino también, lo que es aún más grave, en la clase de pruebas empíricas que invocan para defender sus postulados. A la postre, es muy posible que este debate en torno a las inferencias esté viciado desde su origen, y puede resultar trivialmente cierto que los dos modelos tengan razón. Quizá el problema radica en que se arranca de una falsa dicotomía entre procesos locales y procesos globales que toma estos dos tipos de procesos como categorías discretas e independientes, en lugar de considerar la dimensión local-global como una dimensión continua que discurre entre, por una parte, procesos estrictamente locales y automáticos, que manejan información lingüística y conceptual accesible a bajo coste y operan de continuo, y por otra, procesos de integración global de la información que tienen lugar intermitentemente en momentos singulares del procesamiento.

Un problema de fondo, que no parece interesar mucho a los investigadores sobre las inferencias y que a mi juicio está en la base del falso debate mencionado en el último párrafo, afecta a lo que podríamos llamar la «realidad psicológica» de los procesos de inferencia. Decir que las inferencias son absolutamente necesarias en el procesamiento de textos es casi una tautología. El problema está en que cuando hablamos de inferencias en este terreno no sabemos a ciencia cierta de qué estamos hablando. Por ello, empleamos conceptos y categorías de sentido común, fundamentalmente semánticos, que nos permitan ordenar nuestras intuiciones sobre este campo de conocimiento, con la esperanza de que los procesos cognitivos subyacentes a las operaciones que postulamos se correspondan nítidamente con las categorías que emergen de nuestras intuiciones. Esta es, probablemente la razón de que las tipologías de inferencias sean tan múltiples y variadas. Sin embargo, eso no garantiza que los procesos computacionales que soportan nuestro razonamiento inferencial respeten las distinciones semánticas que nos dicta el sentido común. En otras palabras, no avala la realidad psicológica de tales procesos en cuanto operaciones de cómputo. Aún queda mucho por avanzar en este complejo territorio de la cognición humana. Lo que no está del 
todo claro, empero, es que las herramientas conceptuales y metodológicas de que dispone la psicología cognitiva sean, hoy por hoy, suficientes y adecuadas para acometer con éxito la tarea de comprender cabalmente los procesos de comprensión y recuerdo de relatos.

Para terminar, una última observación. En este artículo he ignorado algunas cuestiones indudablemente pertinentes al problema de las relaciones entre memoria y relato. La más llamativa de estas omisiones hace referencia a la estructura de los relatos como género literario, como forma particular de textos o discursos. Existe la sospecha, probablemente fundada, de que la base temporal y episódica de este género de escritura debe imponer restricciones y abrir posibilidades a las formas de abordar su procesamiento. La implicación de la memoria episódica, sin ir más lejos, debe plantear unos requisitos singulares a los procesos de comprensión de relatos frente a otras clases de textos (por ejemplo, textos expositivos como los del discurso científico). Además, el conocimiento (representado en la memoria semántica) de la estructura de los relatos en sus formas arquetípicas también puede desempeñar un papel crucial en su procesamiento, en comparación con esas otras formas de discurso escrito. Esto debe llevarnos a reflexionar sobre hasta qué punto los procesos cognitivos implicados en la comprensión y el recuerdo de relatos puedan ser cualitativamente distintos de los que se ponen en marcha al comprender y recordar textos no narrativos.

\section{Notas}

${ }^{1}$ La noción de metarrepresentación ha sido utilizada en psicología para denotar aquellos estados mentales del organismo, como los deseos, las creencias y las intenciones en general, que toman como objeto otras representaciones (v.gr. la creencia de que $p$ ). En esta acepción mentalista, el concepto de metarrepresentación es equivalente al de "actitud proposicional». En un sentido más genérico, y por tanto aplicable fuera del territorio de la psicología, una metarrepresentación es, simplemente, una representación de otra representación.

\section{Bibliografía}

BAdDeley, A.D. y Hitch, G.J. (1974): Working memory. En G. Bower (Ed.): Recent Advances in Learning and Motivation, vol. 8. Nueva York: Academic Press [Trad. cast. "Memoria en funcionamiento", en M.V. SEBAStián (Ed.), Lecturas de psicología de la memoria. Madrid: Alianza, 1983].

Bruner, J.A. (1986): Actual Minds, Possible Worlds. Cambridge, MA: Harvard University Press. 


\section{Memoria y relato}

Clark, H.H. y Clark, E.V. (1977): Psychology and Language. Nueva York: Harcourt, Brace and Jovanovich.

Donald, M. (1991): Origins of the Modern Mind. Cambridge, MA: Harvard University Press.

ElosúA, M.R. (2000): Procesos de la comprensión, la memoria y el aprendizaje de textos. Madrid: Sanz y Torres.

ERICSSON, K.A. y Kintsch, W. (1995): Long-term Working Memory. Psychological Review, 102, 211-245.

Gathercole, S.A. y Baddeley, A.D. (1993): Working Memory and Language. Hillsdale, N.J: Lawrence Erlbaum.

Graesser, A.C. y KREUZ, R.J. (1993): A Theory of Inference Generation During Text Comprehension. Discourse Processes, 1-2, 145-160.

KinTSCH, W. y VAN DiJK, T. (1978): Toward a Model of Text Comprehension and Production. Psychological Review, 85, 363-394.

LEÓN, J.A. (Ed.) (2003): Conocimiento y discurso. Madrid: Pirámide.

Leslie, A. (1987): Pretense and Representation: The Origins of "Theory of Mind". Psychological Review, 94, 412-426.

McKoon, G. y RAtCliff, R. (1992): Inference During Reading. Psychological Review, 99, 440-466.

Quine, V. O. (1960): Word and Object. Cambridge, MA: MIT Press. [Trad. cast. Palabra $y$ objeto. Barcelona: Labor, 1968].

Rosa, A., Backhurst, D. y Belleli, G. (Eds.) (2002): Memoria colectiva e identidad nacional. Madrid: Biblioteca Nueva.

Tulving, E. (2002): Memoria episódica y memoria semántica. En R.A. Wilson y F. KeIL (Eds.), Enciclopedia del MIT de ciencias cognitivas. Madrid: Síntesis (vol. 2). 